\title{
Erratum to: Experimental study on heavy ion single-event effects in flash-based FPGAs
}

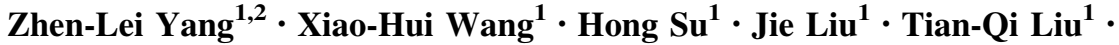 \\ Kai $\mathrm{Xi}^{1,2} \cdot$ Bin Wang ${ }^{1,2} \cdot$ Song Gu ${ }^{1,2} \cdot$ Qian-Shun She ${ }^{1}$
}

Published online: 25 April 2016

(C) Shanghai Institute of Applied Physics, Chinese Academy of Sciences, Chinese Nuclear Society, Science Press China and Springer

Science+Business Media Singapore 2016

\section{Erratum to: NUCL SCI TECH (2016) 27:7 DOI 10.1007/s41365-016-0015-8}

The original version of this article contains some mistakes.

1. The last sentence in the 2nd paragraph in Section 5.1 The logic tiles

The test results indicate that the sequential logic cross section depends not too on the device's operating frequency.

Should be changed into

The test results indicate that the sequential logic cross section depends not too much on the device's operating frequency.
2. The last sentence in Section 5.3 Single-event latch-up The results indicate that the ProASIC 3 product family is not sensitive to SEL.

Should be changed into

The results indicate that the ProASIC 3 product family is not sensitive to SEL up to a LET of $99.0 \mathrm{MeV} \mathrm{cm}$ / mg.

3. Figure 11 should be updated as below (Fig. 11):

The online version of the original article can be found under doi:10.1007/s41365-016-0015-8.

\footnotetext{
Hong $\mathrm{Su}$

suhong@impcas.ac.cn

1 Institute of Modern Physics, Chinese Academy of Sciences, Lanzhou 730000, China

2 University of Chinese Academy of Sciences, Beijing 100049, China
} 


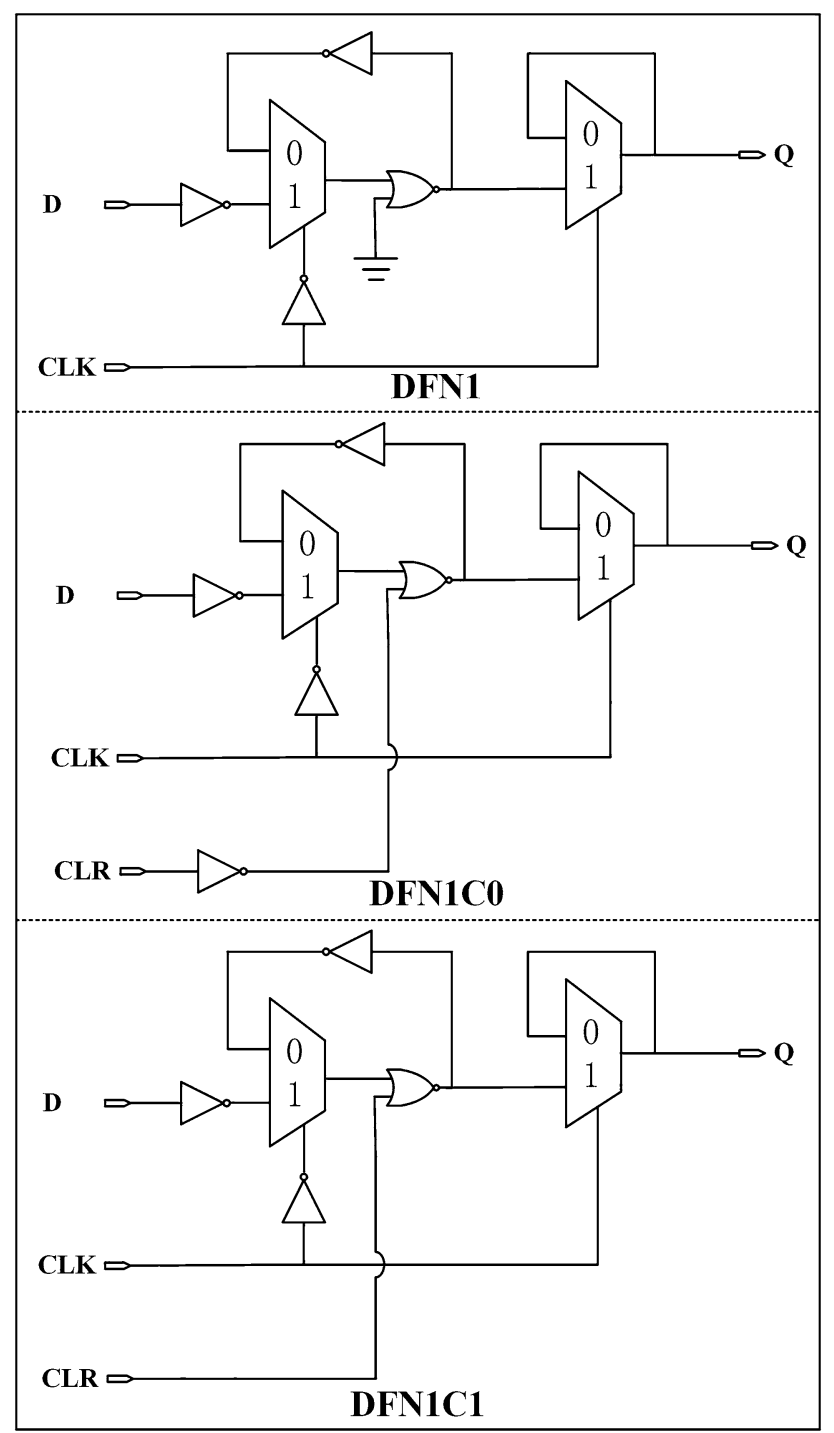

Fig. 11 The simple schematics of the DFN1, DFN1C0 and DFN1C1 implemented on the logic tile 